\title{
Mefenamic Acid for the Prevention of Bleeding and Spotting From Depot-medroxyprogesterone Acetate: A Randomized Controlled Trial
}

\author{
Jen Sothornwit ${ }^{1 *}$, Yuthapong Werawatakul ${ }^{1}$, Orathai Saenbon ${ }^{1}$
}

\begin{abstract}
Objectives: To evaluate the efficacy of mefenamic acid for the reduction of bleeding and spotting in post-partum women initiating the use of depot-medroxyprogesterone acetate (DMPA) for contraception.

Materials and Methods: This double-blind, placebo-controlled study included postpartum breastfeeding women in Khon Kaen, Thailand. Mefenamic acid or placebo was administered over the first 12 weeks of DMPA use. Then, participants completed a self-report bleeding diary.

Results: Forty women, initiating the use of DMPA for postpartum contraception, were randomized to mefenamic acid ( $\mathrm{n}=20)$ or placebo $(n=20)$ group. The study was discontinued after 27 months because of suboptimal enrollment. The mefenamic acid group was less likely to have prolonged bleeding compared to placebo although this was not statistically significant $(37.5 \% \mathrm{vs} .50 \%$, respectively, $\mathrm{P}=0.491)$. DMPA discontinuation rates were high at $50 \%$ in both groups although no participants in the mefenamic acid group stopped using DMPA due to bleeding side effects.

Conclusions: The high discontinuation rates in the postpartum use of DMPA are attributed to the occurrence of bleeding/spotting side effects. Although mefenamic acid prophylaxis appeared to be beneficial to some women, a larger randomized controlled trial is required to confirm the effectiveness of this approach.

Keywords: Breastfeeding, DMPA, Irregular bleeding, Postpartum, Progestin
\end{abstract}

\section{Introduction}

Depot-medroxyprogesterone acetate (DMPA) is an injectable progestin contraceptive. One injection provides pregnancy protection for 13 weeks. This method is highly effective with a $6 \%$ typical use failure rate (1). According to a global report from the United Nations, 74 million women are currently using injectable contraceptive methods (2). However, unscheduled vaginal spotting and bleeding can lead to the discontinuation of progestin contraceptive methods (3). The type and dose of progestin and the duration of use have been shown to play an important role in the severity of bleeding symptoms (3). Up to $90 \%$ of women using DMPA report spotting or bleeding side effects (4), especially after the first to second injection, and unscheduled bleeding/spotting usually persists for more than 7 days (5). Approximately one-third of DMPA users discontinue the medicine within one year (6) and side effects have been cited as one of the main reasons for discontinuation (4).

Unscheduled vaginal bleeding is a common side effect associated with all forms of progestin-only contraceptives. Many plausible etiologies of this side effect have been proposed, including an elevated prostaglandin level, endometrial atrophy, or fragility of the vasculature $(7,8)$. However, the pathogenesis has not been fully elucidated, and as a result, various preventative medications have been studied in clinical trials, and nonsteroidal antiinflammatory drugs (NSAIDs), estrogen, mifepristone, and doxycycline have shown clinical improvements (9). Mefenamic acid is an NSAID that is commonly used in the treatment of pelvic pain which is compatible with breastfeeding (10). This drug has demonstrated effectiveness in the cessation of bleeding among DMPA users (11) although no previous study has evaluated its effectiveness in postpartum women.

The treatment of bleeding in postpartum women is a challenge, particularly because there are concerns that medical therapies containing estrogen may negatively affect breastfeeding (12). Accordingly, the use of medications for the prophylaxis of the irregular bleeding associated with progestin-only contraceptives is worth considering regarding improve satisfaction while decreasing discontinuation rates. The objective of this study was to compare bleeding and spotting in the new users of DMPA for postpartum contraception randomized to either oral mefenamic acid or oral placebo over a 12 week treatment period.

\section{Materials and Methods}

Participants and Setting

This prospective, randomized study was conducted at Srinagarind Hospital, a university hospital in Khon Kaen, 
Thailand between July 2017 and October 2019. Healthy postpartum women over the age of 18 planning to use DMPA after delivery were enrolled and willing to comply with the study protocol. On the other hand, women were excluded if they were diagnosed with uterine abnormalities and coagulopathies or had contraindications to NSAIDs or progestin.

Women were screened for eligibility and enrolled at the time of presentation to the postpartum clinic. After obtaining written informed consent, participants completed the anonymous, self-administered, demographic, and medical information questionnaire. The randomization schedule was created using a computer-based random sequence generator with a $1: 1$ allocation ratio. Participants were then assigned to either the mefenamic acid $500 \mathrm{mg}$ or placebo group by receiving numbered, opaque, and sealed envelopes. Participants were instructed to take the medication twice daily for five days every four weeks for 90 days after DMPA administration. This dosing frequency has been reported in prior studies to reduce progestin-induced bleeding $(11,13,14)$. The mefenamic acid and placebo arms were blinded to the participants and the researchers. A nurse at the family planning clinic, who was not involved in the study, enclosed and distributed the study medications. After recruitment, all participants were requested to record the episodes of bleeding/spotting on a menstrual calendar. Study visits were scheduled at twelve weeks after DMPA administration. At this visit, women returned the diary and completed the questionnaire regarding the side effects, the intensity of breastfeeding, desire to continue DMPA, and satisfaction. Participants were contacted via mobile phone if did not return for their in-person followup visits. In addition, those not attending in person were asked to send a picture of their bleeding diary to a research assistant via the line messaging application and were required to complete the same questionnaire as those who came to the clinic.

\section{Operational Definition}

According to the World Health Organization Belsey criteria regarding bleeding associated with hormonal contraceptives (15), prolonged bleeding is defined as bleeding/spotting episodes lasting longer than 14 days.

\section{Statistical Analysis}

On the basis of a previous study (13), a 27\% difference was estimated in the number of women without prolonged bleeding between the treatment and placebo groups. Hence, 90 women (45 in each group) were needed with a power of $80 \%$, a level of significance of 0.05 , and a $10 \%$ attrition rate.

The primary outcome was the percentage of women who had prolonged bleeding/spotting. Secondary outcomes included the days of bleeding/spotting, side effects of mefenamic acid and DMPA, the continuation of DMPA, and satisfaction. The baseline characteristics of participants in the intervention and control groups were compared using either a chi-squared test or Fisher's exact test. The Student's t-test or the Wilcoxon rank-sum test was used to compare continuous variables between the two groups. Analyses were performed with Stata statistical software, version 12.0 (StataCorp, College Station, TX, USA).

The study was discontinued prior to meeting the sample size goal due to difficulties encountered in enrollment and ethical concerns that arose during the outbreak of coronavirus disease 2019.

\section{Results}

During the enrollment period, 138 women were approached at the family planning clinic. Of these, 40 were eligible and agreed to participate in the study. The average age of participants was $29.1 \pm 4.9$ years old. Among the original 40 participants, we were able to contact 30 women at their 3-month follow-up. Therefore, the number of included subjects in the analysis was 30 made up of 16 and 14 in the mefenamic acid and placebo groups, respectively.

The baseline characteristics of participants in the mefenamic acid and placebo groups are compared in Table 1. The characteristics between the two groups were similar regarding age, body mass index, parity, and the mode of delivery.

Table 2 compares the number of days of bleeding/ spotting and the instances of prolonged bleeding between the two groups. The median days of spotting, days of bleeding, and days of bleeding/spotting appeared to be less in the mefenamic acid group. However, the differences did not reach the significance level. Half of the participants in both groups reported prolonged bleeding during the study period.

The frequency of side effects including weight change, breast tenderness, acne, and dyspepsia was similar between the two groups. For satisfaction scores, a higher proportion of women in the mefenamic acid group reported being satisfied or highly satisfied with the intervention compared to the placebo group (87.5\% vs. $71.4 \%$, respectively). The rate of DMPA continuation, however, was only $50 \%$ in both groups (Table 2 ).

Table 1. Demographics of Study Participants

\begin{tabular}{lcc}
\hline Variables & $\begin{array}{c}\text { Mefenamic Acid Group } \\
(\mathbf{n = 2 0})\end{array}$ & $\begin{array}{c}\text { Placebo Group } \\
(\mathbf{n = 2 0})\end{array}$ \\
\hline Age (years) & $29.2 \pm 5.3$ & $29 \pm 4.8$ \\
$\mathrm{BMI}\left(\mathrm{kg} / \mathrm{m}^{2}\right)$ & $23.3 \pm 2.7$ & $24.1 \pm 4$ \\
Parity & & $18(90)$ \\
1 & $16(80)$ & $2(10)$ \\
$\quad$ More than 1 & $4(20)$ & $13(65)$ \\
Mode of delivery & & $7(35)$ \\
$\quad$ Normal labor & $13(65)$ & \\
Cesarean section & $7(35)$ & \\
\hline Note. BMl: Body mass index. Data are presented as mean \pm standard deviation \\
or number (percentage).
\end{tabular}


Table 2. Bleeding/Spotting Within 90 Days After the Initiation of DMPA, Side Effects, Satisfaction, and Continuation

\begin{tabular}{|c|c|c|c|}
\hline Bleeding/Spotting & Mefenamic Acid Group $(n=16)$ & Placebo Group $(n=14)$ & $P$ Value \\
\hline Days of spotting & $2.5 \pm 6.8$ & $2.5 \pm 6.3$ & 1.000 \\
\hline Days of bleeding & $3.5 \pm 10.8$ & $7.5 \pm 11$ & 0.715 \\
\hline Days of bleeding/spotting & $4 \pm 19.5$ & $13 \pm 22.5$ & 0.272 \\
\hline Number of women with prolonged bleeding & $6(37.5)$ & $7(50)$ & 0.491 \\
\hline \multicolumn{4}{|l|}{ Side effects } \\
\hline Weight reduction $(\mathrm{kg})$ & $2.4 \pm 1.9$ & $1.5 \pm 1.3$ & 0.149 \\
\hline Breast tenderness & $5(31.3)$ & $2(14.3)$ & 0.151 \\
\hline Acne & $3(18.8)$ & $0(0)$ & 0.088 \\
\hline Dyspepsia & $2(12.5)$ & $0(0)$ & 0.186 \\
\hline Satisfaction & & & 0.313 \\
\hline Very satisfied & $4(25)$ & $5(35.7)$ & \\
\hline Satisfied & $10(62.5)$ & $5(35.7)$ & \\
\hline Unsatisfied & $2(12.5)$ & $4(28.6)$ & \\
\hline Very unsatisfied & $0(0)$ & $0(0)$ & \\
\hline Continuation of DMPA & $8(50)$ & $7(50)$ & 1.000 \\
\hline
\end{tabular}

Note. DMPA: Depot medroxyprogesterone acetate. Data are presented as the median \pm the interquatile range or number (percentage).

The primary reason cited for the discontinuation of DMPA was weight gain $(37.5 \%)$ in women receiving mefenamic acid while those receiving placebo reported that bleeding/spotting (42.9\%) was the main reason for the cessation of DMPA. It is noteworthy that no participants in the mefenamic acid group stopped using DMPA due to the side effects of bleeding.

\section{Discussion}

The comparison of mefenamic acid and placebo for the prevention of DMPA-associated bleeding in postpartum women was not feasible in our institution in this randomized controlled trial due to suboptimal enrollment. Nevertheless, mefenamic acid seemed to be beneficial to some women in this cohort. The median number of days of bleeding/spotting was lower (but not significant) in women allocated to the mefenamic acid group. The bleeding side effect was the main reason for the discontinuation of DMPA in the placebo group whereas this reason was not mentioned among discontinuers in the mefenamic acid group. In addition, less than one-fifth of women were unsatisfied with mefenamic acid.

Irregular bleeding due to progestin-only contraception is a common problem, hence, several studies have examined the effectiveness of various agents for treatment and prophylaxis (9). However, no study has been conducted with postpartum women. It was found that the proportion of women with prolonged bleeding was not different from those reported in studies in the general population (16). The effect of mefenamic acid, however, was not as profound as those reported in another therapeutic treatment study (11). For prophylaxis, one trial investigated the influence of NSAIDs (naproxen) in levonorgestrel intrauterine system users (13). The result in this study is consistent with those of a prior study reporting fewer median bleeding/spotting days in women who received naproxen compared to the placebo group during a 90-day reference period although this difference was not significant (27.5 days vs. 32 days, respectively) (13).

The gastrointestinal side effect is one disadvantage of conventional NSAIDs. In the present study, this side effect appeared to be tolerable since few women in the mefenamic acid group reported their experience to be "unsatisfactory" and no women reported "very unsatisfactory", which is in agreement with the findings of a prior study on mefenamic acid (11). Overall, modest postpartum weight loss was detected in our cohort. However, only women in the mefenamic acid group cited weight change as the reason for discontinuation. A plausible explanation for this difference is that those in the placebo group were more concerned with bleeding problems rather than weight change. A recent cohort study demonstrated that the 12-month continuation rate of provider-administered DMPA to be $70.4 \%$ (17), which was extremely higher than the $50 \%$ rate in this study. Surprisingly, no previously published evidence exists regarding the discontinuation rates of DMPA among breastfeeding women.

To promote birth spacing, improving family planning practices in postpartum women is of utmost importance. However, a huge challenge to overcome is the side effects of contraceptive methods in this population. The current trial explored the effectiveness of mefenamic acid to mitigate progestin-associated bleeding side effects, which has not previously been described in the literature. However, this study had some limitations. Suboptimal enrollment precludes us from drawing conclusions. Although differences were observed between the two groups, factors influencing the effect of this intervention it is still unknown. Nevertheless, the results of this study can be used as pilot data for conducting a larger trial. It is believed that a study to identify factors associated with progestin-induced bleeding in post-partum women 
should be conducted to improve decision-making about which women would benefit from prophylactic treatment. Finally, a study in the non-breastfeeding post-partum population would be fascinating and may depict the more profound efficacy of this medication.

\section{Conclusions}

The discontinuation rate of DMPA in postpartum women is high. Side effects including, but not limited to, bleeding/ spotting are the main reasons for discontinuation. Although this study was terminated because of suboptimal enrollment, mefenamic acid prophylaxis seemed to be favorable to some women. Nevertheless, a larger randomized controlled trial is required to confirm the effectiveness of this approach.

\section{Authors' Contribution}

JS and YW conceptualized and designed the study. The manuscript was prepared by JS and revised by YW. OS contributed to the data collection. All authors reviewed and approved the final version of the manuscript.

\section{Conflict of Interests}

Authors declare that they have no conflict of interests.

\section{Ethical Issues}

The approval was obtained from the Ethics Committee of the Institutional Review Board of the Khon Kaen University in Human Research (HE591247). The trial was registered with clinicaltrials.in.th (TCTR20160712001). The applied procedures in this study adhere to the tenets of the Declaration of Helsinki. Additionally, written informed consent was obtained from all participants.

\section{Financial Support}

This work was supported by the Faculty of Medicine, Khon Kaen University, Thailand [IN61119].

\section{Acknowledgments}

Our special gratitude goes to Dr. Glenn Borlace for editing this manuscript via the Publication Clinic, Khon Kaen University, Thailand..

\section{References}

1. Jacobstein R, Polis CB. Progestin-only contraception: injectables and implants. Best Pract Res Clin Obstet Gynaecol. 2014;28(6):795-806. doi:10.1016/j.bpobgyn.2014.05.003

2. United Nations, Department of Economic and Social Affairs, Population Division (2019). Contraceptive Use by Method 2019: Data Booklet (ST/ESA/SER.A/435).

3. Zigler RE, McNicholas C. Unscheduled vaginal bleeding with progestin-only contraceptive use. Am J Obstet Gynecol. 2017;216(5):443-450. doi:10.1016/j.ajog.2016.12.008

4. Chotnopparatpattara P, Taneepanichskul S. Use of depot medroxyprogesterone acetate in Thai adolescents. Contraception.
2000;62(3):137-140. doi:10.1016/s0010-7824(00)00157-8

5. Hubacher D, Lopez L, Steiner MJ, Dorflinger L. Menstrual pattern changes from levonorgestrel subdermal implants and DMPA: systematic review and evidence-based comparisons. Contraception. 2009;80(2):113-118. doi:10.1016/j. contraception.2009.02.008

6. Barden-O'Fallon J, Speizer IS, Calhoun LM, Corroon M. Women's contraceptive discontinuation and switching behavior in urban Senegal, 2010-2015. BMC Womens Health. 2018;18(1):35. doi:10.1186/s12905-018-0529-9

7. White JO, Sullivan $\mathrm{MH}$, Patel L, et al. Prostaglandin production in human endometrium following continuous exposure to lowdose levonorgestrel released from a vaginal ring. Contraception. 1991;43(4):401-412. doi:10.1016/0010-7824(91)90077-s

8. Ovarian and endometrial function during hormonal contraception. Hum Reprod. 2001;16(7):1527-1535. doi:10.1093/ humrep/16.7.1527

9. Abdel-Aleem H, d'Arcangues C, Vogelsong KM, Gaffield ML, Gülmezoglu AM. Treatment of vaginal bleeding irregularities induced by progestin only contraceptives. Cochrane Database Syst Rev. 2013(10):CD003449. doi:10.1002/14651858.CD003449. pub5

10. Chaves RG, Lamounier JA. [Breastfeeding and maternal medications]. J Pediatr (Rio J). 2004;80(5 Suppl):S189-198. doi:10.2223/1246

11. Tantiwattanakul P, Taneepanichskul S. Effect of mefenamic acid on controlling irregular uterine bleeding in DMPA users. Contraception. 2004;70(4):277-279. doi:10.1016/j. contraception.2004.04.003

12. Pieh Holder KL. Contraception and breastfeeding. Clin Obstet Gynecol. 2015;58(4):928-935. doi:10.1097/ grf.0000000000000157

13. Madden T, Proehl S, Allsworth JE, Secura GM, Peipert JF. Naproxen or estradiol for bleeding and spotting with the levonorgestrel intrauterine system: a randomized controlled trial. Am J Obstet Gynecol. 2012;206(2):129.e1-8. doi:10.1016/j.ajog.2011.09.021

14. Kaewrudee S, Taneepanichskul S, Jaisamraun U, Reinprayoon D. The effect of mefenamic acid on controlling irregular uterine bleeding secondary to Norplant use. Contraception. 1999;60(1):25-30. doi:10.1016/s0010-7824(99)00059-1

15. Belsey EM, Machin D, d'Arcangues C. The analysis of vaginal bleeding patterns induced by fertility regulating methods. World Health Organization Special Programme of Research, Development and Research Training in Human Reproduction. Contraception. 1986;34(3):253-260. doi:10.1016/00107824(86)90006-5

16. Jain JK, Nicosia AF, Nucatola DL, Lu JJ, Kuo J, Felix JC. Mifepristone for the prevention of breakthrough bleeding in new starters of depo-medroxyprogesterone acetate. Steroids. 2003;68(1013):1115-1119. doi:10.1016/s0039-128x(03)00132-6

17. Cover J, Ba M, Drake JK, MD ND. Continuation of self-injected versus provider-administered contraception in Senegal: a nonrandomized, prospective cohort study. Contraception. 2019;99(2):137-141. doi:10.1016/j.contraception.2018.11.001

(c) 2021 The Author(s); This is an open-access article distributed under the terms of the Creative Commons Attribution License (http:// creativecommons.org/licenses/by/4.0), which permits unrestricted use, distribution, and reproduction in any medium, provided the original work is properly cited. 